\title{
Calculation of the Excitation Energies of a Hydrogen Impurity in Niobium
}

\author{
F. T. Sommer*, K. Hau, P. Frodl, and F. Wahl \\ Institut für theoretische Physik der Universität Tübingen
}

Z. Naturforsch. 43a, 923 -929 (1988); received December 10, 1987

Recent calculations of the electronic structure of a hydrogen atom in niobium using microscopic first principles are extended in that the dynamical behaviour of a hydrogen impurity centre in $\mathrm{Nb}$ is considered quantummechanically. The calculated excitation energies are in good agreement with experimental values of time of flight-spectra taken from $\mathrm{Nb} \mathrm{H}_{0.05}$.

\section{Introduction}

In the work of Hau, Frodl, Sommer, and Wahl [1] the electronic structure of a single hydrogen impurity centre in Niobium is described. There the Niobium ions and the hydrogen nucleus are treated as classical particles. The displacements of the lattice ions caused by the presence of the impurity centre are described in the frame of classical harmonic theory.

In the present work the consideration of the hydrogen nucleus will be extended to a quantum mechanical treatment which opens the view to the dynamical properties of the impurity centre and allows for the evaluation of excitation energies to be compared with experimental results. We investigate the energy difference between a hydrided infinite niobium crystal, which we call the charged system, and its pure counterpart, the reference system.

\section{Formulation of the Energy Difference}

For the charged system, the quantum mechanical treatment of the crystal electrons and the impurity centre within the classical host lattice yields the many particle Schroedinger equation

$$
\begin{aligned}
{\left[H_{x}^{\mathrm{e}}(\tilde{X})+H_{Y}^{\mathrm{p}}(\tilde{X})\right] } & \Psi_{i j}(x, Y, \tilde{X}) \\
& =E_{i j}(\tilde{X}) \Psi_{i j}(x, Y, \tilde{X}) .
\end{aligned}
$$

\footnotetext{
* Present address: C. u. O.-Vogt Institut für Hirnforschung, Universitätsstr. 1, D-4000 Düsseldorf 1.

Reprint requests to Prof. Dr. F. Wahl, Institut für Theoretische Physik, Universität Tübingen, Auf der Morgenstelle 14, D-7400 Tübingen 1 .
}

$x, Y$ and $\tilde{X}$ in (1.1) are the coordinates of the crystal electrons, the proton and the host lattice ions, respectively. We consider the case $T=0$, i.e. there are no thermally induced lattice ion movements. Further we neglect the deformation of the host lattice caused by the presence of the impurity. As carried through in [2], we apply a twofold adiabatic approximation in (1.1). On the one hand we disregard the influence of the electron and proton motions on the motion of the lattice ions. Thus we make an adiabatic decomposition between the lattice ion motions and the proton and electron motions. Therefore the positions in the undisturbed lattice, $\tilde{X}$, enter (1.1) as a set of fixed values. As potentials of the proton and the lattice ions we use Coulomb potentials, so the Hamiltonians of (1.1) are

$$
\begin{aligned}
H^{\mathrm{e}}(\tilde{X})= & -\sum_{\mu} \frac{\hbar^{2}}{2 m_{\mathrm{e}}} \Delta_{x}+\frac{1}{2} \sum_{\mu, \mu^{\prime}} \frac{e^{2}}{\left|x_{\mu}-x_{\mu^{\prime}}\right|} \\
& -\sum_{\mu} \frac{e^{2}}{\left|x_{\mu}-Y\right|}-\sum_{\mu, n} \frac{Z e^{2}}{\left|x_{\mu}-\tilde{X}_{n}\right|}, \\
H^{\mathrm{p}}(\tilde{X})= & -\frac{\hbar^{2}}{2 m_{\mathrm{p}}} \Delta_{Y}+\sum_{n} \frac{Z e^{2}}{\left|Y-\tilde{X}_{n}\right|} .
\end{aligned}
$$

$Z$ is the charge number of the host lattice ions, $m_{\mathrm{e}}$ and $m_{\mathrm{p}}$ denote the mass of the electron and of the proton.

For the wave function in (1.1) we make the product ansatz

$$
\Psi_{i j}(x, Y, \tilde{X})=\varphi_{i}(x, Y, \tilde{X}) \chi_{i j}(Y, \tilde{X}) .
$$

On the other hand we carry out an adiabatic decomposition between the motions of the electrons and the proton. This means that the electrons are seeing the proton as a classical particle and find themselves in their groundstate relative to the instantaneous pro- 
ton position. This proceeding converts (1.1) in to two coupled equations of electron subsystem and the proton subsystem:

$$
\begin{aligned}
& H^{\mathrm{e}}(\tilde{X}) \varphi_{i}(x, Y, \tilde{X})=R_{i}^{\mathrm{e}}(Y, \tilde{X}) \varphi_{i}(x, Y, \tilde{X}), \\
& \begin{aligned}
{\left[E_{i}^{\mathrm{e}}(Y, \tilde{X})+H^{\mathrm{p}}(\tilde{X})\right] } & \chi_{i j}(Y, \tilde{X}) \\
& =E_{i j}^{\mathrm{p}}(\tilde{X}) \chi_{i j}(Y, \tilde{X})
\end{aligned}
\end{aligned}
$$

The total energy of the charged system becomes

$$
E_{i j}^{\mathrm{H}}=E_{i j}^{\mathrm{P}}\left(\tilde{X}+E_{\mathrm{W}}(\tilde{X}),\right.
$$

where $E_{\mathrm{W}}(\tilde{X})$ is the energy of the classically treated host lattice ions in their ideal lattice positions.

For the reference system we get after an adiabatic decomposition between the crystal electrons and the host lattice ions

$$
H^{\mathrm{R}, \mathrm{e}}(\tilde{X}) \varphi_{k}^{\mathrm{R}}(x, \tilde{X})=E_{k}^{\mathrm{R}, \mathrm{e}}(\tilde{X}) \varphi_{k}^{\mathrm{R}}(x, \tilde{X})
$$

with the Hamiltonian

$$
\begin{aligned}
H^{\mathrm{R}, \mathrm{e}}(\tilde{X})= & -\sum_{\mu} \frac{\hbar^{2}}{2 m_{\mathrm{e}}} \Delta_{x}+\frac{1}{2} \sum_{\mu, \mu^{\prime}} \frac{e^{2}}{\left|x_{\mu}-x_{\mu^{\prime}}\right|} \\
& -\sum_{\mu, n} \frac{Z e^{2}}{\left|x_{\mu}-\tilde{X}_{n}\right|} .
\end{aligned}
$$

The total energy of the reference system is

$$
E_{k}^{\mathrm{R}}=E_{k}^{\mathrm{R} \cdot \mathrm{e}}(\tilde{X})+E_{\mathrm{W}}(\tilde{X}) .
$$

Now we are able to define* the energy difference between the charged system and the reference system in its groundstate $(k=0)$ :

$$
\begin{aligned}
\Delta E:= & E_{i j}^{\mathrm{H}}-E_{0}^{\mathrm{R}} \\
= & \left\langle\chi_{i j}(Y, \tilde{X}),\left[E_{i}^{\mathrm{e}}(Y, \tilde{X})+H^{\mathrm{p}}(\tilde{X})\right]\right. \\
& \left.\cdot \chi_{i j}(Y, \tilde{X})\right\rangle_{Y}-E_{0}^{\mathrm{R}, \mathrm{e}}(\tilde{X}) \\
= & \left\langle\chi_{i j}(Y, \tilde{X}),\left[E_{i}^{\mathrm{e}}(Y, \tilde{X})-E_{0}^{\mathrm{R}, \mathrm{e}}(\tilde{X})+H^{\mathrm{p}}(\tilde{X})\right]\right. \\
& \left.\cdot \chi_{i j}(Y, \tilde{X})\right\rangle_{Y} \\
= & \left\langle\chi_{i j}(Y, \tilde{X}), O_{\mathrm{P}}(\tilde{X}) \chi_{i j}(Y, \tilde{X})\right\rangle_{Y} .
\end{aligned}
$$

The scalar product $\langle\ldots, \ldots\rangle_{Y}$ in $(1.11)$ denotes the integration over the proton coordinates. In the following one has to consider the eigenvalues of the operator

$$
O_{\mathrm{P}}(\tilde{X})=\left[\omega(Y, \tilde{X})+H^{\mathrm{p}}(\tilde{X})\right] .
$$

In (1.12) we used the definition of the electronic energy difference

$$
\omega(Y, \tilde{X}):=E_{i}^{\mathrm{e}}(Y, \tilde{X})-E_{0}^{\mathrm{R}, \mathrm{e}}(\tilde{X}) .
$$

* This definition of the energy difference differs by a constant from that given in [1].

\section{Electronic Energy Difference}

As described in [2], the electronic energy difference in (1.13) will be calculated with the New Tamm Dancoff procedure, wich is a field theoretical method to calculate energy differences. The mathematical structure of the NTD formalism has been investigated by Feist and Wahl in [3] and [4]. Under the transformation in a suitable tensor Fock space the second quantized problems of the charged and the reference system can be reduced to one functional eigenvalue equation. In the lowest approximation, the so called zeroth approximation, the functional eigenvalue equation of the electronic difference problem can be reduced to the equation

$$
\begin{gathered}
S_{1}(z, x)\left[D\left(x, x^{\prime}, \tilde{X}\right)-\frac{e^{2}}{|x-Y|} \delta\left(x-x^{\prime}\right)\right] \\
S_{1}\left(x^{\prime}, y^{\prime}\right) h\left(y^{\prime}\right)=\left(\omega(Y, \tilde{X})-V_{\mathrm{P}, \mathrm{v}}(Y, \tilde{X})\right) h(z) \\
=: \lambda(Y, \tilde{X}) h(z) .
\end{gathered}
$$

In (2.1) and further we use the extended Einstein convention, i.e. one has to integrate multiple arguments.

As discussed in [5], the absolute square of the solution function $|h(x)|^{2}$ can be interpreted as the additional electron density induced by the presence of the impurity centre.

$S_{1}\left(x, x^{\prime}\right)$ is the projection operator on the unoccupied states of the conduction band of the niobium crystal, $D\left(x, x^{\prime}, \tilde{X}\right)$ is the Hartree-Fock operator of the pure crystal. $V_{\text {P.V }}(Y, \tilde{X})$ represents the potential of the embedded proton in the coulomb field of the valence electrons. The eigenvalue $\lambda(Y, \tilde{X})$ of (2.1) enters the proton calculations in paragraph 3 as the additional electronic potential.

The solution of (2.1) in the case of niobium as host crystal has been solved in [1] for the proton fixed at the tetrahedral interstitial site. The Hartree-Fock operator was formulated with a Slater- $X_{\alpha}$ approximation for the exchange interaction as a local operator. Niobium being a transition metal of the electronic configuration $(4 \mathrm{~d})^{4}(5 \mathrm{~s})^{1}$, its electron density is extremely inhomogenious. The expression $V_{\mathrm{P}, \mathrm{V}}(Y, \tilde{X})$, which also occurs in the Hartree-Fock operator as potential of the valence electrons, can be constructed with a valence electron density function $n_{\mathrm{e}}(r)$ :

$$
V_{\mathrm{V} . \mathrm{P}}(Y, \tilde{X})=-e^{2} \int \frac{n_{e}\left(r^{\prime}\right)}{\left|Y-r^{\prime}\right|} \mathrm{d} r^{\prime} .
$$


This density function $n_{\mathrm{e}}(r)$ of the niobium valence electrons was originally calculated in [6] by means of selfconsistent pseudopotential calculations and represented in [7] in polar coordinates by the use of spherical functions. For the expansion of the operators $S_{1}\left(x, x^{\prime}\right)$ of equation (2.1) in [1] only the plane wave states describing the $5 \mathrm{~s}$-valence electrons were used. The so constructed projection operator is only orthogonalizing on the $5 \mathrm{~s}$-valence states. A consideration of the $4 \mathrm{~d}$-valence states in the construction of $S_{1}\left(x, x^{\prime}\right)$ would lead to an additional repulsive effect in the proximity of the lattice ions. A similar repulsive effect results if we take into consideration the spatial extension of the Niobium ion caused by the presence of its core electrons. In [1] these effects are reproduced by introducing a repulsive correction potential which is added to the simple coulomb potential of the lattice ions. For this additional ionic potential the ansatz

$$
V_{\mathrm{HC}}(r)=\frac{Z e^{2}}{r} \mathrm{e}^{-\gamma x^{2}}
$$

is made. The parameter $\gamma$ governs the range of the additional ionic hardcore ${ }^{* *}$. In [1], a range is given in which the hardcore parameter $\gamma$ should be situated: $0.4 \leq \gamma \leq 0.5$. We use in the present calculations $\gamma=0.4$, which will be subject to discussion in the last part of this paper.

In [1], as a set of expansion functions for $h(x)$ the hydrogen functions $\phi_{n l m}^{z}\left(r^{\prime}\right)$ to the ionic charge numbers $Z=1$ and $Z=5$ are used. Additionally, these functions have been cut below the Fermi energy by applying the projection operator $S_{1}\left(x, x^{\prime}\right)$, so that the condition $h(x)=S_{1}\left(x, x^{\prime}\right) h\left(x^{\prime}\right)$ holds.

$$
h(r)=\beta_{0} \psi_{100}^{1}(r)+\sum_{n, l, m} \beta_{n l m} \psi_{n l m}^{5}(r)
$$

with

$$
\psi_{n l m}^{z}(r)=S_{1}\left(r, r^{\prime}\right) \phi_{n l m}^{z}\left(r^{\prime}\right) .
$$

For the problem of the proton being displaced from the tetrahedral interstitial site we use the expansion system (2.4). Different from previous works, [8] and [9], the expansion functions are centered at the instantaneous place of the proton and not at the interstitial site. The potential around the interstitial site plotted

\footnotetext{
** To simplify the further discussion we talk about the ionic hardcore although the additional potential is of Gaussian type and not a hardcore.
}

in [1] shows a strong dominance of the unscreened proton potential compared to the lattice ions potential, which is screened by the inhomogenious valence electron density. The wave function of the additional electron density $h(x)$ is faced by a potential for which the potential of the Jellium model is no bad approximation. In the Jellium model the Coulomb potentials of the lattice ions and of the inhomogenious valence electron density are just compensating each other. In that model we expect of course a complete translation invariance relative to the instantaneous proton coordinate. We used this fact to check the numerical convergence of a interstitial centered expansion system. Jellium model calculations in which the expansion sum was carried to $n=7$ and $l=3$ were far away from reproducing this translation invariance of the proton. Carrying on the expansion to $l=4$, which represents the limit of the tenable numerical effort at the moment, didn't improve that result. This fact lead us to the choice of an expansion system which follows the proton at each moment. If we separate the proton coordinate: $Y=\tilde{Y}+y$, where $\tilde{Y}$ is the coordinate of the interstitial place, the expansion system can be written

$$
h_{y}^{\prime}(r):=\beta_{0} \psi_{100}^{1}(r-y)+\sum_{n l m} \beta_{n l m} \psi_{n l m}(r-y) .
$$

Insertion of (2.6) into (2.1) and transformation of the coordinates origin in the instantaneous proton place (which is possible because of the translation invariance of $\left.S_{1}\left(x, x^{\prime}\right)\right)$ leads to

$$
\begin{gathered}
S_{1}\left(x, x^{\prime}\right)\left[D\left(x^{\prime}+y, \tilde{X}\right)+\frac{e^{2}}{\left|x^{\prime}\right|}\right] S_{1}\left(x^{\prime}, z\right) h(z) \\
=\lambda(y) h(x),
\end{gathered}
$$

where $h(x)$ is the interstitial centered expansion system (2.4).

The expansion of $h(x)$ is carried out to $n=7$ and $l=3$ which reduces $(2.7)$ to a $79 \times 79$ matrix equation for the coefficients $\beta_{n l m}$ in (2.4). The solution of this equation yields the additional electronic potential $\lambda(y)$ required in the protonic calculations on a point lattice.

As it can be seen in Fig. 2, the additional electron potential $\lambda(y)$ bears an antibinding character. We are able to state at this point that an increase of $\gamma$, which implies a reduction of the hardcore range, leads to a reinforcement of the antibinding character of the additional electron potential $\lambda(y)$. 


\section{Protonic Subsystem}

On the grid on which $\lambda(x)$ is given, a numerical calculation of the eigenvalues of the operator $O_{\mathrm{P}}$ of (1.12) is possible. With (2.1) equation (1.12) becomes

$$
\begin{aligned}
O_{\mathrm{P}}(\tilde{X}) & =\left[H^{\mathrm{P}}(\tilde{H})+V_{\mathrm{P}, \mathrm{V}}(Y, \tilde{X})+\lambda(y, \tilde{X})\right] \\
& =\left[-\frac{\hbar^{2}}{2 m_{\mathrm{p}}} \Delta_{y}+G(y, \tilde{X})\right],
\end{aligned}
$$

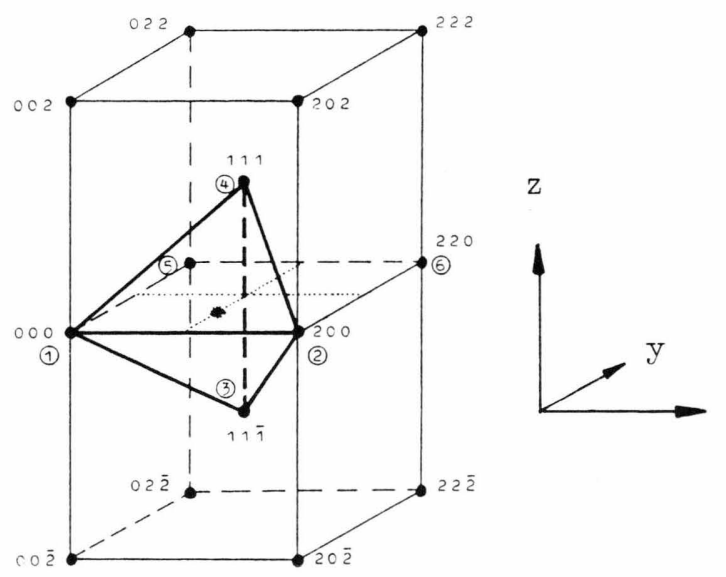

Fig. 1. Tetrahedral interstitial site in a bcc lattice. where with (2.2) and (1.3) the effective adiabatic proton potential is given by

$$
\begin{aligned}
G(y, \tilde{X}):= & \sum_{n} \frac{Z e^{2}}{\left|y-\tilde{X}_{n}\right|} \\
& -e^{2} \int \frac{n_{\varepsilon}\left(r^{\prime}\right)}{\left|y-r^{\prime}\right|} \mathrm{d} r^{\prime}+\lambda(y, \tilde{X}) .
\end{aligned}
$$

For the further evaluation of the second expression in (3.2) it is necessary to consider the explicit form of the valence electron density used in [7], which was originally calculated in [6] for pure $\mathrm{Nb}$ by the use of pseudo potential calculations.

$$
n_{\varepsilon}(\boldsymbol{r})=\frac{Z}{V_{0}}\left[\sum_{n} n^{\prime}\left(\boldsymbol{r}-\tilde{\boldsymbol{X}}_{n}\right)+n_{0}\right]
$$

with

$$
n^{\prime}(\boldsymbol{r})= \begin{cases}n_{1}(r)+n_{2}(r) W(\vartheta, \varphi)-n_{0} & \text { for } r \leq R_{i} \\ 0 & \text { for } r>R_{i}\end{cases}
$$

$R_{i}$ is the half distance from one lattice ion to its nearest neighbour, $V_{0}$ denotes the volume of the Wigner-Seitz cell and $(r, \vartheta, \varphi)$ is the representation of $\boldsymbol{r}$ in polar coordinates.

In the second expression of (3.2) it is possible to split the integral over the whole crystal volume into a sum of integrals on each Wigner-Seitz cell. So we can ex-
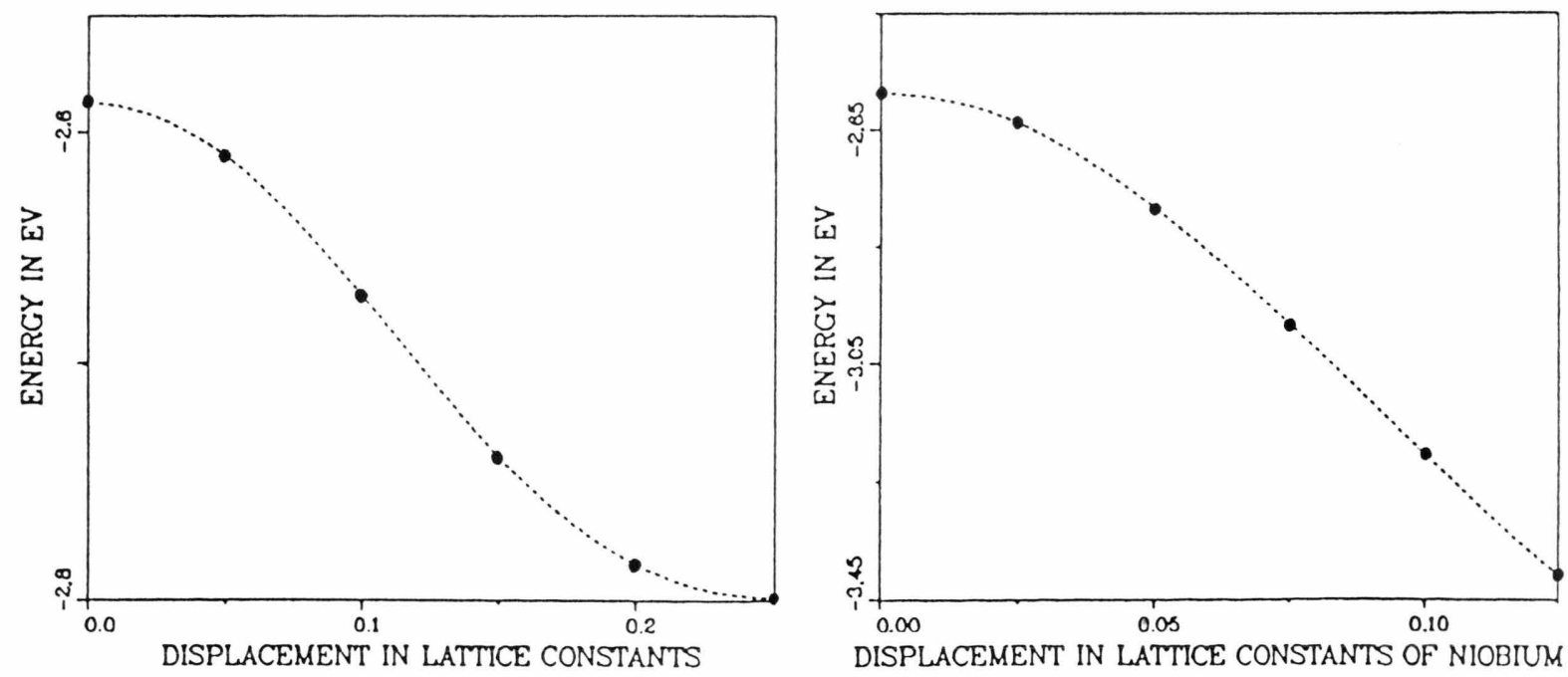

Fig. 2. Diagrams of the additional electronic potential $\lambda(y, \tilde{X})$ in $y$ direction (left) and $x$ direction (right). Orientation of the displacement directions in the lattice see Figure 1. 
press the first two terms in (3.2):

$$
\begin{aligned}
P(y):= & \sum_{n} \frac{Z e^{2}}{\left|y-\tilde{X}_{n}\right|}-e^{2} \int \frac{n_{\varepsilon}\left(r^{\prime}\right)}{\left|y-r^{\prime}\right|} \mathrm{d} r^{\prime} \\
= & \sum_{n} e^{2}\left[\frac{Z}{\left|y-\tilde{X}_{n}\right|}-\frac{Z n_{0}}{V_{0}} \int_{\mathbf{V}_{0}} \frac{1}{\left|y-\tilde{X}_{n}-r^{\prime}\right|} \mathrm{d}^{2} r^{\prime}\right] \\
& -\underbrace{\sum_{n} \frac{Z e^{2}}{V_{0}} \int_{\mathbf{V}_{0}} \frac{n^{\prime}\left(r^{\prime}\right)}{\left|y-\tilde{X}_{n}-r^{\prime}\right|} \mathrm{d}^{3} r^{\prime} .}_{=: V_{n^{\prime}(r)}}
\end{aligned}
$$

$V_{n^{\prime}(r)}$ represents the potential resulting from the inhomogenious contribution $n^{\prime}(r)$ of the valence electron density. The lattice sum in $V_{n^{\prime}(r)}$ can be separated into two parts. For lattice ions situated in the proximity of the proton we evaluate exactly the integral over $n^{\prime}(r)$. The valence electron density near far away lattice ions is taken into account as negative virtual point charges $Z_{v}$ located in the ionic positions. In the following it is more suitable to sum up the far away approximation over the whole lattice and to add a correction term for the lattice ions in the proximity of the proton:

$$
\begin{aligned}
P(y)= & \underbrace{\sum_{n} e^{2}\left[\frac{Z-Z_{v}}{\left|y-\tilde{X}_{n}\right|}-\frac{Z n_{0}}{V_{0}} \int_{\mathbf{V}_{0}} \frac{1}{\left|y-\tilde{X}_{n}-r^{\prime}\right|} \mathrm{d}^{3} r^{\prime}\right]}_{=P_{\mathrm{F}}(y)} \\
& +\underbrace{\sum_{n, N} e^{2}\left[\frac{Z_{v}}{\left|y-\tilde{X}_{n}\right|}-\frac{Z}{V_{0}} \int_{\mathbf{V}_{0}} \frac{n^{\prime}\left(r^{\prime}\right)}{\left|y-\tilde{X}_{n}-r^{\prime}\right|} \mathrm{d}^{3} r^{\prime}\right]}_{=P_{\mathbf{K}}(y)} .
\end{aligned}
$$

The notation n.N means "nearest neighbours". How many nearest neighbours have to be chosen in order to get a good approximation, depends on the spatial structure of the valence electron density $n_{\varepsilon}(r)$. This can be estimated after the treatment of the correction term $P_{\mathbf{K}}(y)$.

For the far away approximation term we obtain after Fourier expansion

$$
P_{\mathrm{F}}(y)=\frac{4 \pi Z n_{0} e^{2}}{V_{0}} \sum_{k \neq 0} \frac{e^{i \boldsymbol{k} \boldsymbol{y}}}{k^{2}} .
$$

The convergence of the reciprocal lattice sum being slow, we use the convergence acceleration method of Ewald-Fuchs [10].

In the correction term we reparate $n^{\prime}(\boldsymbol{r})$ into a radial by symmetric part and a contribution which is depending on the polar coordinate angle of $\boldsymbol{r}$. The potential of the radial by symmetric part can be expressed exactly by use of virtual point charges at the ionic sites. After some transformations [11], the correction term becomes

$$
\begin{aligned}
& P_{\mathrm{K}}(y)= \\
& \quad-e^{2} \frac{Z}{V_{0}} \frac{8 \pi^{2}}{45} \sum_{n . N} \frac{1}{\left|y-\tilde{X}_{n}\right|^{5}} \Theta\left(\omega_{y-\tilde{X}_{n}}\right) \int_{0}^{R_{i}} n_{2}\left(r^{\prime}\right) r^{\prime 6} \mathrm{~d} r^{\prime}
\end{aligned}
$$

with the angle function $\Theta\left(\omega_{y-\tilde{X}_{n}}\right)$, a function depending only on the polar coordinate angle $\omega_{y-\tilde{X}_{n}}$ of the vektor $y-\tilde{X}_{n}$. The function $\Theta\left(\omega_{y-\tilde{X}_{n}}\right)$ is explicitly given in [11].

The structure of $n_{\varepsilon}(r)$ yields an expression for $P_{\mathrm{K}}(\mathrm{y})$, in which the proton-ion distance enters the denominator in the fifth power. Hence we get a fast convergence, and even in summing over only a small number of neighbour ions we obtain $P_{\mathrm{K}}(y)$ with good accuracy. In the present calculations we used the ions within a radius of four lattice constants around the occupied interstitial site.

The diagrams in Fig. 3 show the entire potential of the proton operator in directions of the $x$-, $z$-axis and the $y$-axis. A displacement of the proton in the $y$-direction provides the maximum possible distance of the proton from its nearest neighbours. In this direction we expect the potential wall separating the proton from the next interstitial place to be lowest. This potential wall should be significant for diffusion properties like the diffusion coefficient. The solution of the eigenvalue equation (the dependence on the lattice coordinates will be suppressed furtheron)

$$
O_{\mathrm{P}} \chi_{i j}(\boldsymbol{y})=E_{i j} \chi_{i j}(\boldsymbol{y})
$$

can be executed with the product ansatz

$$
\chi_{i j}(\boldsymbol{y})=\chi_{x}^{i}\left(y_{x}\right) \chi_{y}^{i}\left(y_{y}\right) \chi_{z}^{i}\left(y_{z}\right),
$$

where $y_{x}, y_{y}$ and $y_{z}$ denote the components of the vector $\boldsymbol{y}$. The separation of the three dimensional problem is achieved by using the perturbation theoretical approach

$$
O_{\mathrm{P}}=O_{\mathrm{P}}^{0}+O_{\mathrm{P}}^{1} .
$$

The contribution which can be separated exactly is

$$
\begin{aligned}
O_{\mathrm{P}}^{0} & =-\frac{\hbar^{2}}{2 m_{\mathrm{p}}} \Delta_{y}+G_{x}\left(y_{x}\right)+G_{y}\left(y_{y}\right)+G_{z}\left(y_{z}\right) \\
& =O_{x}^{0}+O_{y}^{0}+O_{z}^{0}
\end{aligned}
$$

with $G_{x}\left(y_{x}\right):=G\left(\left(y_{x}, 0,0\right)\right)$ and $O_{x}^{0}:=-\frac{\hbar^{2}}{2 m_{\mathrm{p}}} \Delta_{y_{x}}$
$+G_{x}\left(y_{x}\right)$ a.s.o. 

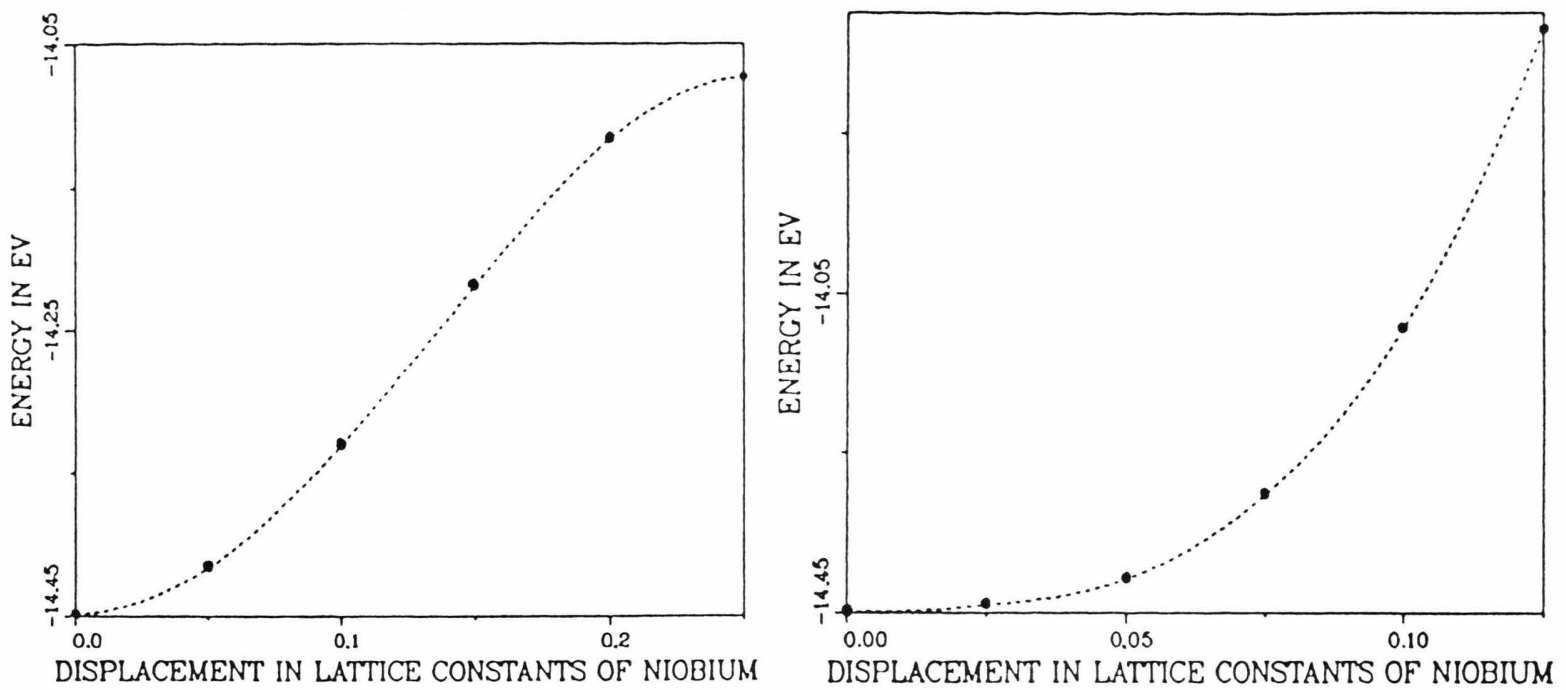

Fig. 3. Diagrams of the entire adiabatic proton potential in $y$ direction (left) and $x$ direction (right).

As inseparable contribution remains

$$
O_{\mathrm{P}}^{1}=G(\boldsymbol{y})-\left[G_{x}\left(y_{x}\right)+G_{y}\left(y_{y}\right)+G_{z}\left(y_{z}\right)\right] .
$$

Under consideration of perturbation terms of the first order we obtain the energy eigenvalues of (3.6)

$$
E_{i j}=E_{i j}^{0}+E_{i j}^{1}
$$

where $E_{i j}^{0}$ equals the sum of the eigenvalues from the one dimensional problems. For instance, the problem in the direction of the $x$-axis can be written as

$$
O_{x}^{0} \chi_{x, l}\left(y_{x}\right)=E_{x, l}^{0} \chi_{x, l}\left(y_{x}\right) .
$$

The energy correction of the first order is

$$
E_{i j}^{1}=\left\langle\chi_{i j}(\boldsymbol{y}), \quad O_{\mathrm{P}}^{1} \chi_{i j}(\boldsymbol{y})\right\rangle_{Y} .
$$

The one dimensional problems can be solved numerically on the abscissae on which the entire proton potential is given. The three dimensional integration in $E_{i j}^{1}$ is also evaluated numerically on the grid on which the proton potential is available. We calculate the ground-state energy and the energies of the three lowest excitation states. The electron subsystem remains always in its ground-state. Each of the three excited states posesses an excitation quantum in one direction of the three axes. Because of the symmetry properties of the bcc lattice of niobium the excitation states in $x$ - and $z$ direction are energy degenerated.

As results we obtain the excitation energy in $x$-, $z$-direction: $185.69 \mathrm{meV}$ (experimental value: $180 \mathrm{meV}$ ) and in $y$-direction: $124.41 \mathrm{meV}$ (experimental value: $110 \mathrm{meV}$ ). The energy correction of the first order amounts for the ground-state to $6.2 \%$, for the excited state in $x-, z$-direction to $4.5 \%$ and for the excited state in $y$ direction to $14.6 \%$. The experimental values are results of neutron scattering experiments which where carried out by Verdan et al. [12].

The above results are quantitatively not completely satisfactory; hence we should analyse all insufficiencies involved. There is the hardcore parameter $\gamma$ as one feature in the niobium model we used, which is not completely fixed. With $\gamma=0.4$ we took the lowest value of the $\gamma$ range suggested in [1]. Regarding the discussion at the end of paragraph 2 concerning the influence of $\gamma$ on the electronic potential, it can be concluded that an increase of $\gamma$ would cause a diminution of the protonic excitation energies. Hence the increase of $\gamma$ could improve the agreement with the experiment. This also confirms the results of [1] where the best agreement with experimental values of the storage energy and lattice deformation was achieved with $\gamma=0.45^{*}$. On the whole the introduction of the ionic correction potential in the underlying niobium model is not quiet satisfactory and should be replaced by a more qualified treatment of the $4 \mathrm{~d}$ valence electrons.

* Because the results of [1] where not known when the present calculations were started, we could not take into account those results in the choice of $\gamma$. 
Another improvement would be a calculation including effects caused by lattice displacements. It will be easy to threat displacements of the nearest neighbours and next nearest neighbours by using the dipole approximation which was applied in [1]. A more accurate calculation of the electronic problem should start from a higher approximation of the NTD-procedure, as it is worked out in [5] and [13].

Diffusion problems of hydrogen in niobium have been successfully investigated by Teichler et al. [14]

[1] K. Hau, P. Frodl, F. Sommer, and F. Wahl, Z. Naturforsch. 43a, 914 (1988).

[2] F. Wahl, R. Duscher, K. Göbel, and J. K. Maichle, Z. Naturforsch. 39a, 524 (1984).

[3] W. Feist, Z. Naturforsch. 36a, 421 (1981).

[4] F. Wahl and W. Feist, Z. Naturforsch. 36a, 429 (1981).

[5] Klaus Göbel and F. Wahl, Z. Naturforsch. 41 a, 1381 (1986).

[6] K. H. Ho, S. G. Louie, J. R. Chelikowsky, and M. L. Cohen, Phys. Rev. B 15, 1755 (1977).

[7] Klaus Hau, Ein Wasserstoffzentrum in Niob: Elektronenstruktur und Gitterstatik, Dissertation Tübingen 1987.

[8] B. Bratschek, Statik und Dynamik eines Zwischengitteratoms im Metallkristall, Diploma work Tübingen 1984. using phenomenological proton potentials. With the knowledge of the adiabatic proton potential resulting from the methods described in this paper a first principles calculation of diffusion properties should be possible and will be subject of following work.

The numerical calculation was executed in Fortran 77 on a BASF $7 / 88$ of the Rechenzentrum der Universität Tübingen.

[9] J. Maichle, Elektronen- und Gitterstruktur eines Wasserstoffzentrums im hexagonalen Metallkristall, Diploma work Tübingen 1983.

[10] A. Haug, Theoretische Festkörperphysik I, Paragraph 4, Springer, Berlin 1961.

[11] F. Sommer, Berechnung von Anregungsenergien lokaler Moden in hydridisiertem Niob, Diploma work Tübingen 1987.

[12] G. Alefeld and J. Völkl, Hydrogen in Metals II, Springer, Berlin 1978.

[13] Manfred Gnirss, Dissertation Tübingen.

[14] A. Klamt and H. Teichler, phys. stat. sol. (b) 134, 103 (1986). 